\title{
Fracture in a thin film of nanotwinned copper
}

\author{
Zhi Zeng ${ }^{1}$, Xiaoyan $\mathrm{Li}^{2}$, Lei $\mathrm{Lu}^{3}$ and Ting $\mathrm{Zhu}^{1 *}$ \\ ${ }^{1}$ Woodruff School of Mechanical Engineering, \\ Georgia Institute of Technology, Atlanta, Georgia 30332, USA \\ ${ }^{2}$ Center for Advanced Mechanics and Materials, Applied Mechanics Laboratory, Department of \\ Engineering Mechanics, Tsinghua University, Beijing 100084, China \\ ${ }^{3}$ Shenyang National Laboratory for Materials Science, Institute of Metal Research, \\ Chinese Academy of Sciences, Shenyang 110016, China
}

\begin{abstract}
Nanotwinned $\mathrm{Cu}$ exhibits an unusual combination of ultra-high strength and high tensile ductility. However, its fracture behavior and associated microscopic mechanisms remain largely unexplored. Here we study the fracture in a free-standing thin film of nanotwinned $\mathrm{Cu}$ using molecular dynamics (MD) simulations. For a pre-crack inclined to the twin boundary, MD simulations show a characteristic fracture mode of zigzag cracking, which arises due to periodic deflections of the crack path by twin boundaries. The mechanism of fracture involves the screw dislocation-mediated local thinning ahead of the crack, instead of cleavage fracture. Importantly, MD simulations show a unique fracture footprint of $\langle 110\rangle$-oriented crack edges, consistent with the previous experimental observation from in situ transmission electron microscopy. Our results reveal the toughening mechanisms by nanotwins and also have broader implications for understanding the mechanical failure of metallic thin films.
\end{abstract}

Keywords: nanotwinned $\mathrm{Cu}$, fracture, molecular dynamics, dislocation-induced thinning *Email: ting.zhu@me.gatech.edu 


\section{Introduction}

Ultrafine grained copper with embedded nanoscale twin lamellas (hereafter referred to as nanotwinned $\mathrm{Cu})$ exhibits an unusual combination of ultra-high strength $(\sim 1 \mathrm{GPa})$ and high tensile ductility ( 14\% elongation to failure) [1-4]. However, most engineering applications would require the materials to have high fracture resistances as well [5]. Unlike the strength and tensile ductility that have been extensively studied [6-14], the fracture behavior and associated microscopic mechanisms in nanotwinned metals are much less known.

Several recent studies have investigated the fracture in nanotwinned metals. Qin et al. [15] processed bulk coarse-grained $\mathrm{Cu}$ samples by dynamic plastic deformation and obtained a composite microstructure of nanoscale grains with embedded nanoscale twin bundles. They measured an enhanced fracture toughness relative to samples without nanotwins, and found that twin bundles caused the elongated deep dimples on fracture surfaces that contributed to an increase of fracture energy. Singh et al. [16] measured the fracture toughness and fatigue crack growth in nanotwinned $\mathrm{Cu}$ processed by electrodeposition, and showed that the presence of nanotwins enhanced both monotonic and cyclic crack growth resistances. Shan et al. [17] and Kim et al. [18] used the in situ transmission electron microscopy (TEM) to directly observe crack growth in nanotwinned $\mathrm{Cu}$. Figure 1 shows the representative in situ TEM results of crack growth across twin groups in a thin foil of nanotwinned $\mathrm{Cu}$ [17]. It is seen from Fig. 1(a) that a characteristic zigzag crack path formed during tensile loading. Figure 1(b) presents magnified images of region 1 in Fig. 1(a) showing the $\langle 110\rangle_{\mathrm{M}}$ and $\langle 110\rangle_{\mathrm{T}}$ crack edges in the adjoining matrix (M) and twin (T) crystals. Kim et al. [18] and Zhou and Qu [19] performed molecular dynamics (MD) simulations of fracture in thick nanotwinned samples. They observed the damage and fracture processes, including crack blunting through dislocation emission, crack 
bridging by twin lamella, crack deflection by grain boundaries, and nanovoid formation at the intersections between grain boundaries and twin boundaries.

The experimental and modeling studies discussed above suggest that the crack-twin boundary interactions can be beneficial to enhance fracture toughness. But the toughening mechanism associated with nanotwins are still little understood. Particularly, the following questions have hitherto not been well addressed: What are the intrinsic fracture mechanisms in nanotwinned metals? How do twin boundaries affect crack propagation? The present work is motivated by previous in situ TEM observations $[17,18]$ and aims to address the above questions by studying the growth of a pre-crack in a free-standing thin film of nanotwinned $\mathrm{Cu}$ using MD simulations. Furthermore, MD simulations can directly reveal the underlying atomic-level processes of deformation and fracture, thereby shedding light onto the failure mechanisms of nanotwinned metals.

\section{Method}

Figure 2 shows the setup of MD simulations containing a free-standing thin film of nanotwinned $\mathrm{Cu}$ with a pre-crack. The MD sample has an in-plane size of $22.1 \mathrm{~nm} \times 43.6 \mathrm{~nm}$ and a thickness of $1.7 \mathrm{~nm}$. The twin boundaries are equally separated by $8.6 \mathrm{~nm}$. Each twin lamella consists of the perfect Face-Centered Cubic (FCC) lattice. A pre-crack with a length of $\sim 7 \mathrm{~nm}$ is created at the sample edge by removing a single layer of atoms on the inclined $\{111\}$ plane. As a

result, the pre-crack has its edge along the $\langle 112\rangle_{\mathrm{M}}$ direction in the matrix (M) crystal. The total number of atoms in the system is 146,783 . The periodic boundary condition is imposed only in the horizontal [112] direction. A uniaxial tensile load is applied in [112] direction at a constant strain rate $\dot{\varepsilon}$ of $2 \times 10^{9} / \mathrm{s}$, while both the [110] and [111] directions are traction free. The system 
temperature is maintained at $5 \mathrm{~K}$. We perform MD simulations with an embedded atom method (EAM) potential of $\mathrm{Cu}$ [20] using LAMMPS [21].

\section{Results and Discussion}

Figures 3 presents a sequence of MD snapshots showing the simulated zigzag mode of crack growth. As the applied load increases, dislocations on the $\{111\}_{\mathrm{M}}$ slip plane in the matrix crystal emit from the crack tip and then pile up against the twin boundary that temporarily obstruct the movement of dislocations. As a result, a high local stress arises to act on the leading dislocation in the pileup array. As the applied load continues to increase, this local stress becomes so high that the leading dislocation transmits into the adjoining twin lamella and further glides on the conjugate $\{111\}_{\mathrm{T}}$ slip plane in next twin lamella. Dislocations behind the leading one in the pileup array repeat this "stop-and-go" process. Meanwhile, transmitted dislocations in the adjoining twin lamella develop a pileup array, due to obstruction of the twin boundary further away from the crack tip. A similar "stop-and-go" process occurs at this twin boundary. Gliding of dislocations in each twin lamella causes local thinning at the active slip planes that eventually leads to lamella fracture. Since the crack is periodically deflected by twin boundaries, a zigzag crack path develops in MD simulations, which is consistent with the previous experimental observation through in situ TEM [17], as shown in Fig. 1(a).

To understand the fracture mechanism of zigzag cracking, we note that in the 2D projected view of the film (Fig. 2), the $\{111\}$ pre-crack has its edge along the $\langle 112\rangle_{\mathrm{M}}$ direction. We choose to create such $\{111\}$ pre-crack in order to facilitate cleavage fracture on the close-packed $\{111\}$ planes. However, it is unexpected to observe in Fig. 3 that the edges of the growing crack in MD simulations are primarily aligned with the $\langle 110\rangle$ directions in all lamellas. This footprint of 
$\langle 110\rangle$ crack edges indicates that fracture does not occur by cleavage of $\{111\}$ planes, which otherwise would produce crack edges along the $\langle 112\rangle$ directions.

A detailed analysis of MD results reveals that fracture occurs through a process of dislocation-mediated local thinning instead of cleavage. This is a unique mode of mechanical failure of thin films in the absence of out-of-plane constraints. More specifically, Fig. 4(a) shows a 3D view of the atomic configuration of a partially cracked film. Figures 4(b1) and (b2) respectively expose the (111) cross sections of the film that are cut at two different locations along the crack path, such that the onset and growth of the local thinning can be clearly seen. As schematically shown in Fig. 4(c), the local thinning is mediated by dislocation glide. That is, an extended dislocation, which splits into the leading and trailing partials in FCC $\mathrm{Cu}$, can glide on the conjugate $\{111\}_{\mathrm{M}}$ and $\{111\}_{\mathrm{T}}$ slip planes in the adjoining matrix and twin crystals. Such type of dislocations have the Burgers vector $\mathbf{b}=\langle 110\rangle / 2$ with the non-zero component in the thickness direction of the film. As a result, gliding of those dislocations on $\{111\}_{\mathrm{M}}$ and $\{111\}_{\mathrm{T}}$ slip planes causes the decrease of film thickness, responsible for the local thinning and final rupture of the film. Because of symmetry, the in-plane tensile loading along the [112] direction can activate dislocations on all the equivalent inclined $\{111\}_{\mathrm{M}}$ and $\{111\}_{\mathrm{T}}$ slip planes symmetric about the (110) plane of the film, which eventually produce $\{111\}_{\mathrm{M}}$ and $\{111\}_{\mathrm{T}}$ crack surfaces, as illustrated in Fig. 4(c). Within each twin lamella, the two inclined $\{111\}$ crack surfaces (indicated respectively by a solid and a dashed line) intersect to create an apparent $\langle 110\rangle$ crack edge. Those characteristic $\langle 110\rangle$ crack edges have already been seen in the $2 \mathrm{D}$ projected view of the fractured film in Fig. 1(b) and Fig. 3(d). 
We further analyze the nature of active dislocations responsible for the thinning and fracture of nanotwinned $\mathrm{Cu}$ thin films. Fig. 5 shows a MD snapshot where several dislocations glide in between the upper and lower twin boundaries. The red parallelogram indicates an inclined $\{111\}$ slip plane containing an extended dislocation (between two red curves) that splits into the leading and trailing partials enclosing a stacking fault ribbon in between. This is a screw dislocation with the Burgers vector $\mathbf{b}=\langle 110\rangle / 2$, as illustrated on the schematic Thompson tetrahedron in Fig. 5 . Such type of screw dislocation occurs most frequently in MD simulations. This is because these screw dislocations are subjected to the largest resolved shear stress (corresponding to the largest Schmid factor of 0.41$)$ among the twelve possible $\{111\}\langle 110\rangle$ slip systems in the twin lamella [13], given the in-plane tensile load parallel to the twin boundary. From the standpoint of deformation compatibility, the Burgers vectors of these screw dislocations (i.e., $\mathbf{b}=\langle 110\rangle / 2$ ) have non-zero components in both the in-plane loading direction and the out-of-plane thickness direction. As a result, gliding of these dislocation near the crack tip can produce in-plane elongation to accommodate the applied tensile load and meanwhile reduce film thickness to enable the relaxation of out-of-plane stresses. Moreover, gliding of a screw dislocation on the active $\{111\}_{\mathrm{M}}$ (or $\{111\}_{\mathrm{T}}$ ) slip plane can produce a relative shear displacement between two adjacent $\{111\}_{\mathrm{M}}$ (or $\{111\}_{\mathrm{T}}$ ) atomic planes. As more screw dislocations glide on this active $\{111\}_{\mathrm{M}}$ slip plane, the shear displacement gradually increases and eventually produces a shearoff mode of fracture on the $\{111\}_{\mathrm{M}}$ (or $\{111\}_{\mathrm{T}}$ ) slip plane as illustrated in Fig. 4(c). In addition, we note that the Burgers vectors of these screw dislocations (i.e., $\mathbf{b}=\langle 110\rangle / 2$ ) are parallel to the intersection between the conjugate $\{111\}_{\mathrm{M}}$ and $\{111\}_{\mathrm{T}}$ slip planes at twin boundary, thus facilitating the slip transmission of screw dislocations across twin boundary. 
The TEM images in Fig. 1 also show an intriguing characteristic of fracture path which features several short crack edges on twin boundaries, as highlighted by circles. Since the inplane tensile load was primarily parallel to twin boundaries, the resolved normal and shear stresses on twin boundaries were small. As a result, there was a lack of direct driving forces to produce those short crack edges on twin boundaries. To understand the origin of those crack edges, we note that in both the in situ TEM experiment by Shan et al. [17] and the earlier studies of fracture of thin metal foils [22], it has been found that small-sized nanocracks frequently formed in front of the main crack; and as the tensile load increased, nanocracks grew and coalesced with the main crack. To explore the possible effect of crack coalescence on the formation of crack edges at twin boundaries, we setup two pre-cracks on the two opposite edges of a nanotwinned thin film, as shown in Fig. 6(a). Under an in-plane tensile load parallel to twin boundaries, the two edge cracks grow in the opposite direction, producing zigzag crack paths, similar to the case of a single pre-crack in Fig. 3. Interestingly, as the two cracks meet at a twin boundary, a small ligament typically arises in between two crack tips; in experiments, such meeting with an offset between the two crack tips is conceivably more common than the exact meeting without an offset. As shown by a sequence of MD snapshots in Fig. 6(b-d), the final crack coalescence occurs through rotation and tearing of this small ligament, thereby producing a pair of short crack edges on the twin boundary as circled in Fig. 6(d). Hence, this MD simulation not only reproduces the formation of short crack edges on twin boundaries as observed in the TEM image of Fig. 1(b), but also demonstrates that MD simulations can help clarify the unexpected features and thus reveal the associated microscopic mechanisms during deformation and fracture. 
The above MD simulation results also reveal several toughening mechanisms by nanotwins. First, twin boundaries can obstruct the movement of dislocations emitted from the crack tip. The obstructed dislocations shield the crack tip from the applied load by producing back stresses near the crack tip [23]. As a result, the applied load has to increase to drive the dislocation emission and crack extension processes. Second, due to the periodic deflections of the crack path by twin boundaries, fracture occurs through zigzag cracking. Compared to a straight crack path, the zigzag mode of crack growth increases the effective crack length and thus causes more energy dissipation through irreversible plastic processes near the zigzag crack faces. Third, twin boundaries are coherent and thus have higher fracture resistances than the typical incoherent grain boundaries in nanocrystalline metals [2]. Figure 6 provides an example of fracture at the twin boundary through plastic tearing, which can cause more energy dissipation than the brittle fracture of weak incoherent boundaries. Fourth, the high density and accordingly small spacing of twin boundaries in nanotwinned metals can further enhance the aforementioned toughening mechanisms.

Finally, we comment on the effect of sample thickness on the fracture of nanotwinned metals. When the thickness of nanotwinned $\mathrm{Cu}$ thin films is around $100 \mathrm{~nm}$ or above, previous experimental and computational studies [18] have shown that a large number of dislocations on different $\{111\}\langle 110\rangle$ slip systems were emitted from the crack tip and they interacted with twin boundaries ahead of the crack. As a result, a high density of dislocations were accumulated on twin boundaries, transforming a clean and coherent twin boundary into a dislocation wall with increasing resistances to slip transmission. The dislocation wall effectively resisted crack propagation, such that either the crack was arrested by a twin boundary or the nanoscale twins became crack bridging ligaments. In this work, the nanotwinned sample is very thin. As a result, 
the type of active dislocation slip systems and the interaction between dislocations are significantly restrained, resulting in the operation of a dominant type of screw dislocations as shown in Fig. 5. The persistent slip of these dislocations leads to local thinning and crack extension through twin boundaries.

\section{Conclusions}

To conclude, our molecular dynamics simulations have revealed the zigzag mode of cracking in a free-standing thin film of nanotwinned $\mathrm{Cu}$, which arises due to periodic deflections of the crack by twin boundaries. The microscopic mechanism of fracture involves the screw dislocation-mediated local thinning ahead of the crack, instead of cleavage of close-packed $\{111\}$ planes. The screw dislocation-induced thinning and fracture result in a unique footprint of the $\langle 110\rangle$-oriented crack edges as shown by both the present molecular dynamics simulations and the previous in situ TEM experiments [17]. Our results reveal the toughening mechanisms by nanotwins, including the twin boundary obstruction of dislocation glide, twin boundary deflection of crack path, strong fracture resistance of coherent twin boundary, and high twin boundary density to enhance the aforementioned toughening mechanisms. While the present study is focused on the nanotwinned thin film of $\mathrm{FCC} \mathrm{Cu}$, the fracture mechanism of dislocationmediated thinning is expected to prevail during the mechanical failure of other types of thin FCC metal films as well. The combined MD and (previous) in situ TEM studies provide strong support for the operation of such fracture mechanism. Mechanistic insights gained in this work could be also valuable for studying fracture in nanotwinned crystals with other types of lattice structure [24-26]. 


\section{Acknowledgements}

We acknowledge the support by the NSFC grant 51420105001. TZ acknowledges the support by the NSF grant DMR-1410331. XL acknowledges the support by the NSFC grant 11372152. LL acknowledges the financial support from the National Basic Research Program of China (973 Program, 2012CB932202) and the financial support of the "Hundreds of Talents Project" by the Chinese Academy of Sciences. We thank Chris Eberl and Zhiwei Shan for valuable discussions.

\section{References}

[1] L. Lu, Y.F. Shen, X.H. Chen, L.H. Qian, K. Lu. Ultrahigh strength and high electrical conductivity in copper, Science 304 (2004) 422-426.

[2] K. Lu, L. Lu, S. Suresh. Strengthening Materials by Engineering Coherent Internal Boundaries at the Nanoscale, Science 324 (2009) 349-352.

[3] T. Zhu, H.J. Gao. Plastic deformation mechanism in nanotwinned metals: An insight from molecular dynamics and mechanistic modeling, Scripta Materialia 66 (2012) 843-848.

[4] I.J. Beyerlein, X. Zhang, A. Misra. Growth Twins and Deformation Twins in Metals, Annual Review of Materials Research 44 (2014) 329-363.

[5] E. Bitzek, J.R. Kermode, P. Gumbsch. Atomistic aspects of fracture, International Journal of Fracture 191 (2015) 13-30.

[6] Z.H. Jin, P. Gumbsch, E. Ma, K. Albe, K. Lu, H. Hahn, H. Gleiter. The interaction mechanism of screw dislocations with coherent twin boundaries in different face-centred cubic metals, Scripta Materialia 54 (2006) 1163-1168.

[7] T. Zhu, J. Li, A. Samanta, H.G. Kim, S. Suresh. Interfacial plasticity governs strain rate sensitivity and ductility in nanostructured metals, Proceedings of the National Academy of Sciences of the USA 104 (2007) 3031-3036.

[8] L. Lu, M. Dao, T. Zhu, J. Li. Size dependence of rate-controlling deformation mechanisms in nanotwinned copper, Scripta Materialia 60 (2009) 1062-1066.

[9] L. Lu, Zhu, T., Shen, Y.F., Dao, M., Lu, K., Suresh, S. Stress relaxation and the structure size-dependence of plastic deformation in nano-twinned copper, Acta Materialia 57 (2009) 5165-5173.

[10] L. Lu, X. Chen, X. Huang, K. Lu. Revealing the Maximum Strength in Nanotwinned Copper, Science 323 (2009) 607-610.

[11] X.Y. Li, Y.J. Wei, L. Lu, K. Lu, H.J. Gao. Dislocation nucleation governed softening and maximum strength in nano-twinned metals, Nature 464 (2010) 877-880.

[12] Y.J. Wei. The kinetics and energetics of dislocation mediated de-twinning in nano-twinned face-centered cubic metals, Materials Science and Engineering A 528 (2011) 1558-1566.

[13] Z. You, X. Li, L. Gui, Q. Lu, T. Zhu, H. Gao, L. Lu. Plastic anisotropy and associated deformation mechanisms in nanotwinned metals, Acta Materialia 61 (2013) 217-227. 
[14] Y.M. Wang, F. Sansoz, T. LaGrange, R.T. Ott, J. Marian, T.W. Barbee, Jr., A.V. Hamza. Defective twin boundaries in nanotwinned metals, Nature Materials 12 (2013) 697-702.

[15] E.W. Qin, L. Lu, N.R. Tao, J. Tan, K. Lu. Enhanced fracture toughness and strength in bulk nanocrystalline $\mathrm{Cu}$ with nanoscale twin bundles, Acta Materialia 57 (2009) 6215-6225.

[16] A. Singh, L. Tang, M. Dao, L. Lu, S. Suresh. Fracture toughness and fatigue crack growth characteristics of nanotwinned copper, Acta Materialia 59 (2011) 2437-2446.

[17] Z.W. Shan, L. Lu, A.M. Minor, E.A. Stach, S.X. Mao. The effect of twin plane spacing on the deformation of copper containing a high density of growth twins, JOM 60 (2008) 71-74.

[18] S.-W. Kim, X. Li, H. Gao, S. Kumar. In situ observations of crack arrest and bridging by nanoscale twins in copper thin films, Acta Materialia 60 (2012) 2959-2972.

[19] H. Zhou, S. Qu. The effect of nanoscale twin boundaries on fracture toughness in nanocrystalline Ni, Nanotechnology 21 (2010) 035706.

[20] Y. Mishin, M.J. Mehl, D.A. Papaconstantopoulos, A.F. Voter, J.D. Kress. Structural stability and lattice defects in copper: Ab initio, tight-binding, and embedded-atom calculations, Physical Review B 63 (2001) 224106.

[21] S. Plimpton. Fast Parallel Algorithms For Short-Range Molecular-Dynamics, Journal of Computational Physics 117 (1995) 1-19.

[22] S.M. Ohr. An electron-microscope study of crack tip deformation and its impact on the dislocation theory of fracture, Materials Science and Engineering 72 (1985) 1-35.

[23] T. Zhu, W. Yang, T. Guo. Quasi-cleavage processes driven by dislocation pileups, Acta Materialia 44 (1996) 3049-3058.

[24] T. Zhu, J. Li. Ultra-strength Materials, Progress in Materials Science 55 (2010) 710-757.

[25] Y.T. Zhu, X.Z. Liao, X.L. Wu. Deformation twinning in nanocrystalline materials, Progress in Materials Science 57 (2012) 1-62.

[26] J. Wang, Z. Zeng, C.R. Weinberger, Z. Zhang, T. Zhu, S.X. Mao. In situ atomic-scale observation of twinning-dominated deformation in nanoscale body-centred cubic tungsten, Nature Materials 14 (2015) 594-600. 
(a)

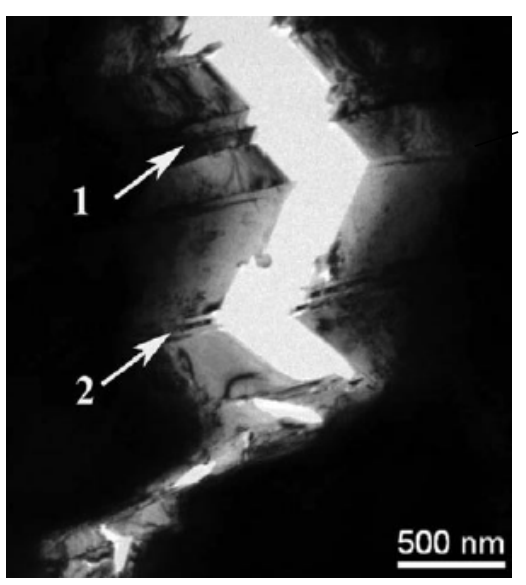

(b)

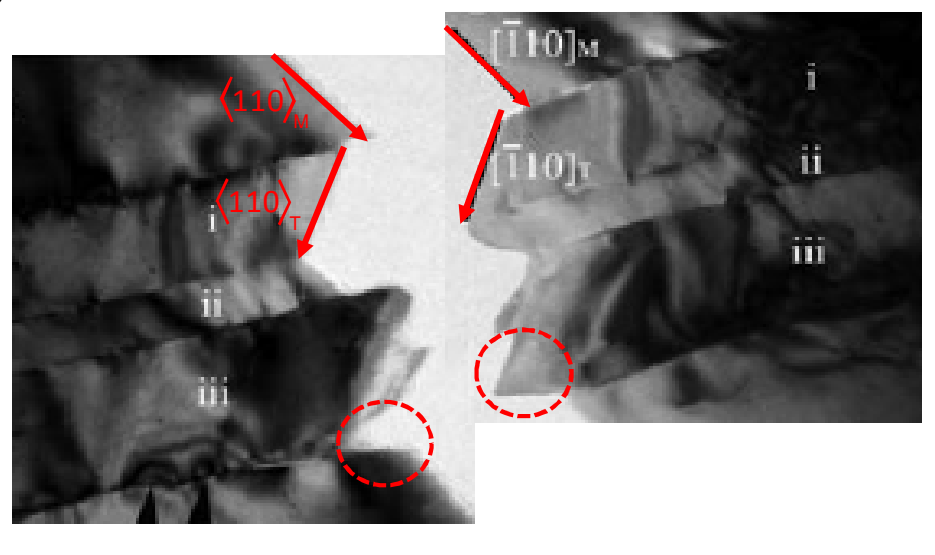

Fig. 1 In situ TEM images of crack growth across twin groups in a thin foil of nanotwinned $\mathrm{Cu}$, from a previous study by Shan et al. [17]. (a) A zigzag crack formed during tensile loading. (b) Magnified images of region 1 in (a), showing the $\langle 110\rangle_{\mathrm{M}}$ and $\langle 110\rangle_{\mathrm{T}}$ crack edges in the adjoining matrix (M) and twin (T) crystals. Circles indicate the short crack edges on twin boundaries. 


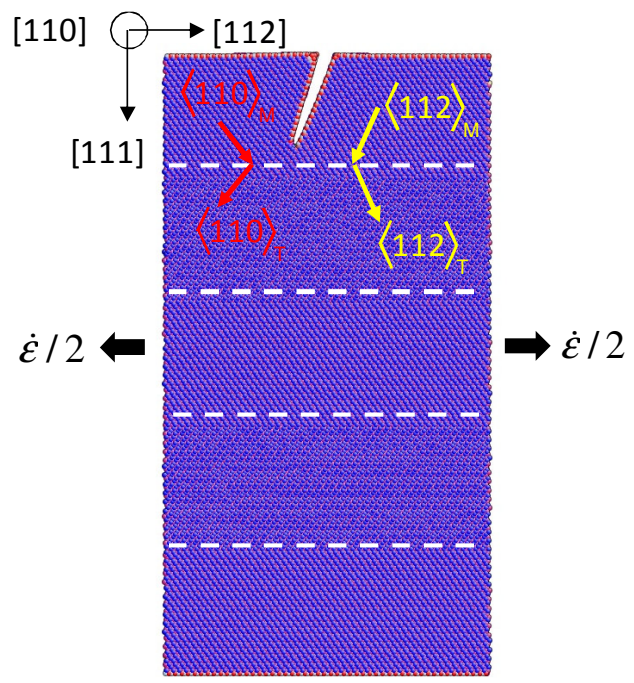

Fig. 2 The MD setup involving a nanotwinned $\mathrm{Cu}$ thin film with a pre-existing edge crack. The dashed lines indicate twin boundaries. A uniaxial tensile load with constant strain rate $\dot{\varepsilon}$ is applied parallel to the twin boundary. Atoms are colored by the coordination number $(\mathrm{CN})$. Atoms in a perfect FCC lattice have $\mathrm{CN}=12$ (yellow), while defective atoms have $\mathrm{CN}=10$ (green), 9 (pink), 8 (white), or 7 (blue). 
(a)

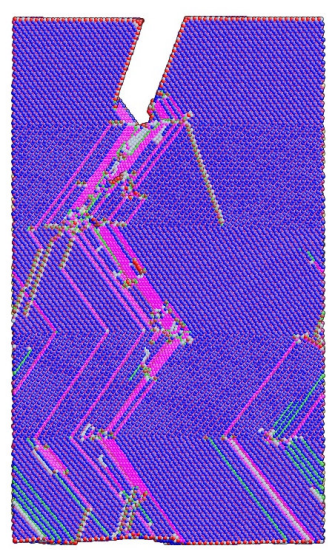

(b)

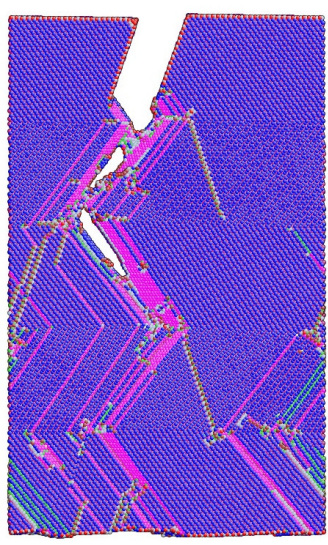

(c)

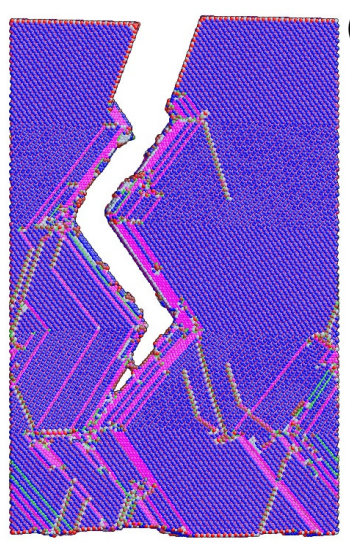

(d)

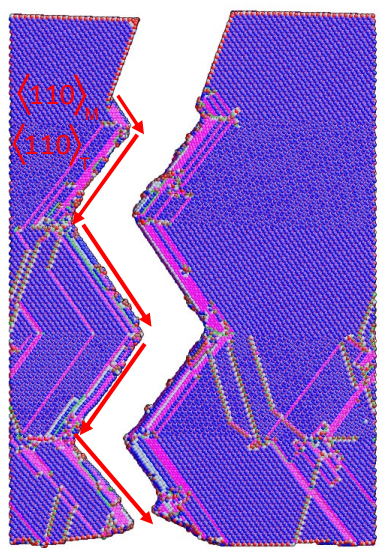

Fig. 3 MD snapshots showing the simulated zigzag mode of crack growth. The applied tensile strains in (a) to (d) are $13.5 \%, 16.3 \%, 20.9 \%$ and $25 \%$, respectively. The edges of the growing crack are along the $\langle 110\rangle_{\mathrm{M}}$ and $\langle 110\rangle_{\mathrm{T}}$ directions in the matrix (M) and twin (T) crystals, respectively. Atoms are colored with the same scheme as in Fig. 2. 
(a)

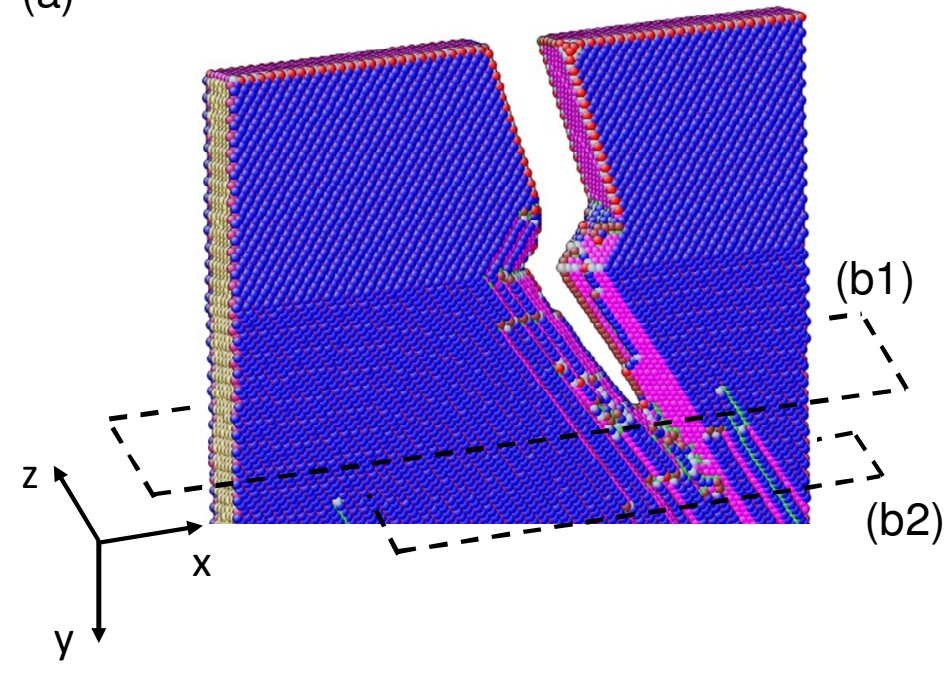

(c)

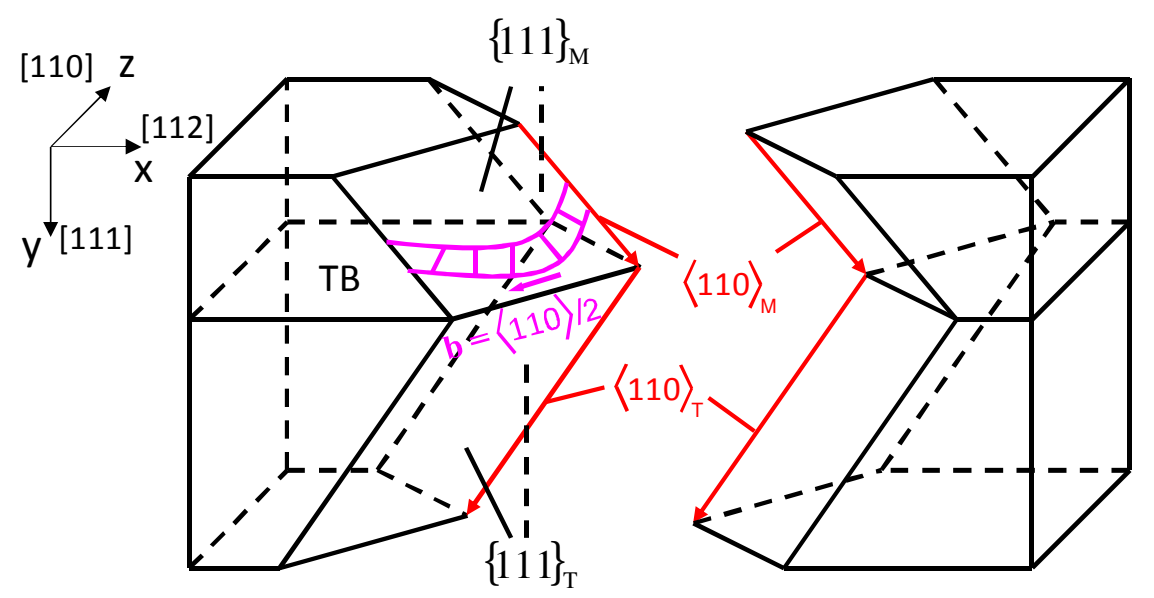

(b1)

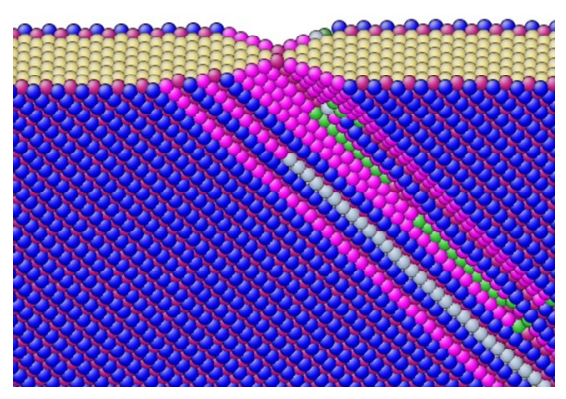

(b2)

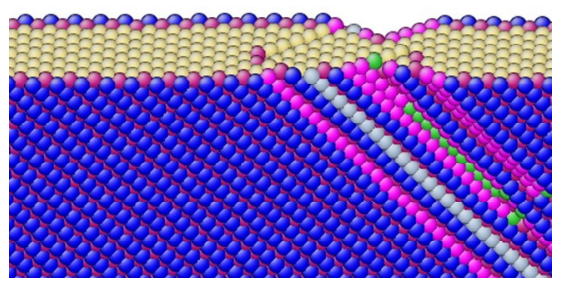

Fig. 4 Fracture via dislocation-mediated local thinning in a thin film of nanotwinned $\mathrm{Cu}$. (a) MD snapshot of a propagating crack. (b) The (111) cross sections are exposed to reveal the onset (b1) and growth (b2) of local thinning at two different locations along the crack extension path. Atoms are colored with the same scheme as in Fig. 2. (c) 3D schematic showing the active mode of dislocation glide as well as the resultant crack surfaces and edges. Dislocations dominantly glide on the inclined $\{111\}$ slip planes, so as to cause the local shear thinning and produce the $\{111\}_{\mathrm{M}}$ and $\{111\}_{\mathrm{T}}$ crack surfaces. Within each twin lamella, the two inclined $\{111\}$ crack surfaces (indicated respectively by a solid and a dashed line) intersect to create the $\langle 110\rangle$ crack edge (indicated by the red arrow). 


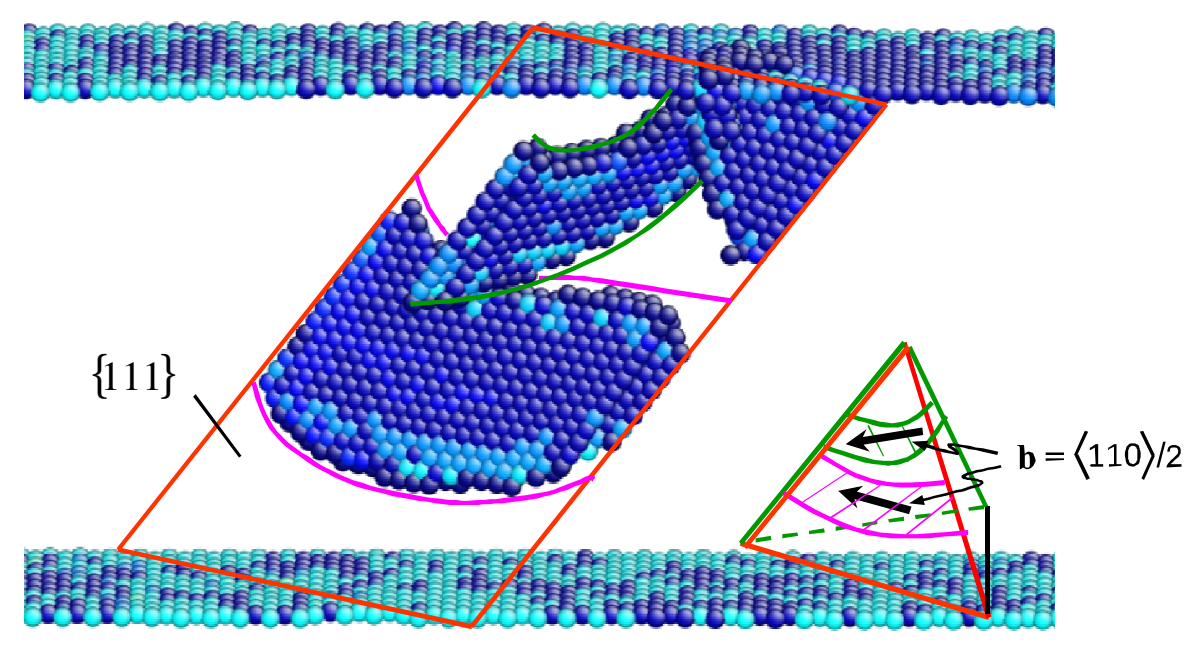

Fig. 5 A MD snapshot showing the active screw dislocations on the $\{111\}\langle 110\rangle$ slip systems, as illustrated by the Thompson tetrahedron. Atoms are colored by the central symmetry parameter, showing the twin boundary and the stacking fault of screw dislocation. 
(a)

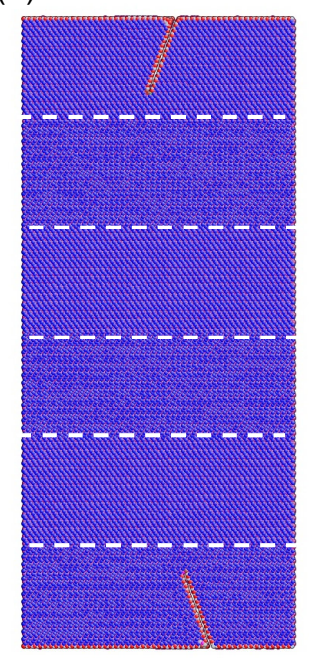

(b)

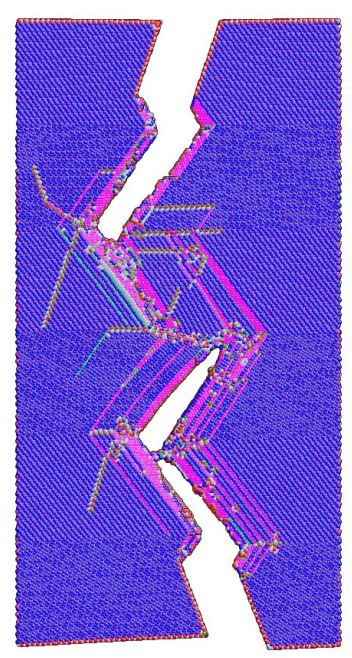

(c)

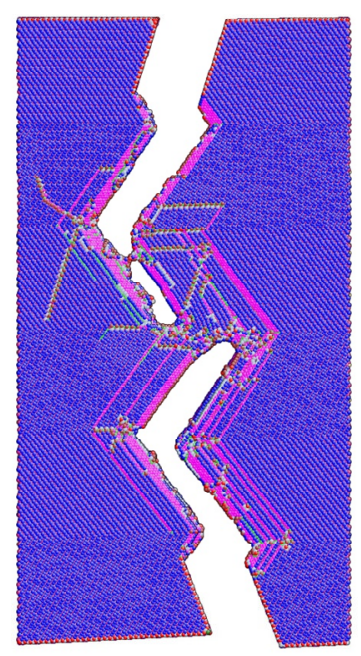

(d)

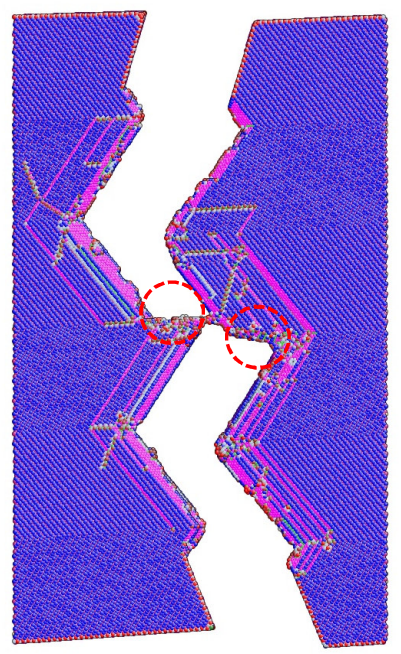

Fig. 6 MD snapshots showing the formation of short crack edges on twin boundaries. (a) MD setup containing two pre-existing edge cracks. The dashed lines indicate twin boundaries. (b-d) A sequence of atomic structures showing the process of crack coalescence, involving the local rotation and tearing of a small ligament between the two crack tips meeting at a twin boundary. The applied tensile strains in (b) to (d) are 20.3\%, 23.7\%, and 38.5\%, respectively. Atoms are colored with the same scheme as in Fig. 2. 

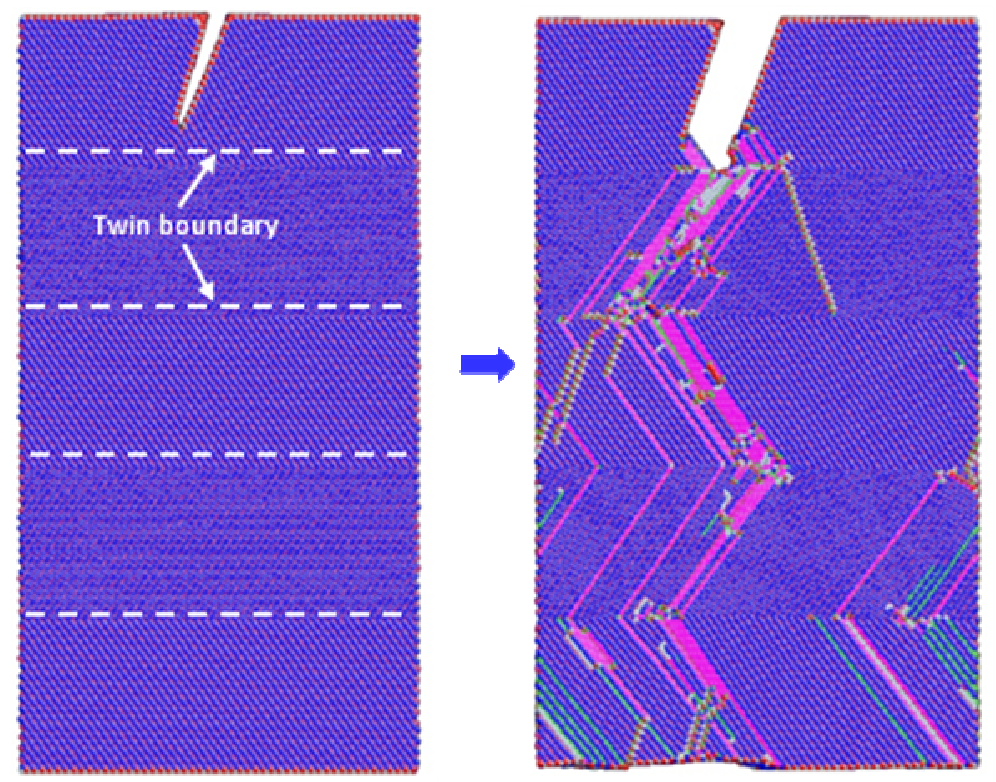

Nanotwinned Cu thin film

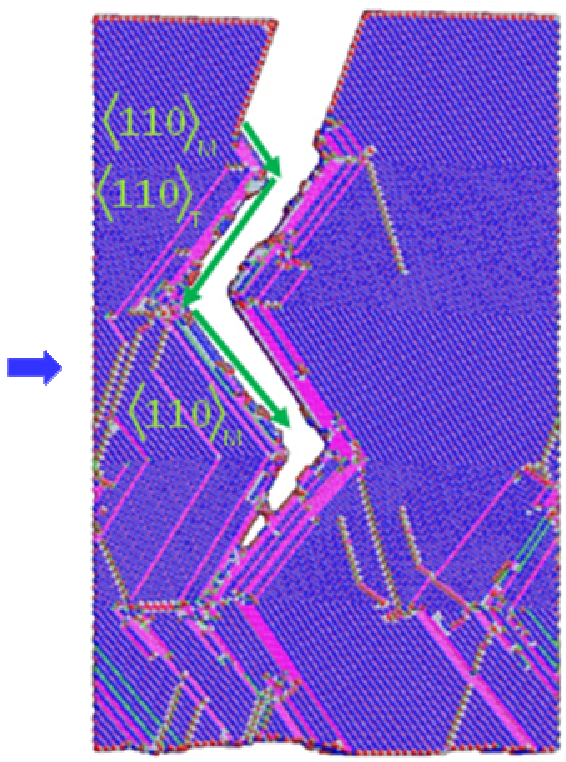

Zigzag cracking with $<110>$ crack cdges 